\title{
An unusual case of aortic valve obstruction
}

\author{
Wilhelm P. MISTIAEN ${ }^{1,2}$, MD, ScD, PhD; Leen UYTTEBROEK ${ }^{2}$, MSc; Guy HUBENS ${ }^{2}, \mathrm{MD}, \mathrm{PhD}$; \\ Luc VAN NASSAUW ${ }^{2}, \mathrm{MSC}, \mathrm{PhD}$
}

${ }^{1}$ Dept. of Healthcare Sciences, Artesis University College of Antwerp, Antwerp, Belgium; ${ }^{2}$ Dept. of Antwerp Surgical Training, Anatomy and Research Centre (ASTARC), Laboratory of Human Anatomy and Embryology, Faculty of Medicine and Health Sciences, University of Antwerp, Belgium.

Summary During a dissection class for anatomy, a white lipoid mass was found in the ascending aorta, which was partly attached to the wall and filled the sinuses of Valsalva and almost fitting as a cast. This mass prevented full opening of the mobile aortic valve leaflets, thereby causing an obstruction. Microscopic analysis revealed fibres and presence of polymorphonuclear white blood cells. It seems reasonable to assume that this mass has formed in the last weeks or months of the life of this subject, which is much quicker than for calcified aortic valve stenosis. Therefore, signs and symptoms of aortic obstruction might have been missed or misinterpreted. In case of timely detection during life, diagnostic imaging and therapeutic approach can be challenging.

Keywords Lipoid aortic valve obstruction.

\section{INTRODUCTION}

Obstruction of the outlet of the ventricle is mainly due to degenerative aortic valve disease, in which infiltration of lipids, inflammation and ossification play an important role. This is the most prevalent heart valve disease in Western societies. In its final stage, the prognosis is poor without aortic valve replacement ${ }^{1,2}$. This case report describes the obstruction of the left ventricular outlet by another cause.

\section{CASE}

During dissection in an anatomy class, the aorta of the corpse of a 62-year-old woman was opened. The only retrievable clinical data indicated that she had died

\section{Address for correspondence:}

Wilhelm P. Mistiaen, Artesis University College of Antwerp, Dept. of Healthcare Sciences, J. De Boeckstraat 9, 2610 Antwerp, Belgium.

E-mail:wilhelm.mistiaen@artesis.be

Received 25 March 2013; accepted for publication 18 April 2013. at home, suddenly. The ascending aorta was filled with a compact white-grayish material (figure 1). The material filled almost the entire aortic root and ascending aorta. It was attached to the aortic wall at its left side, leaving only a small part as outlet for the blood flow.

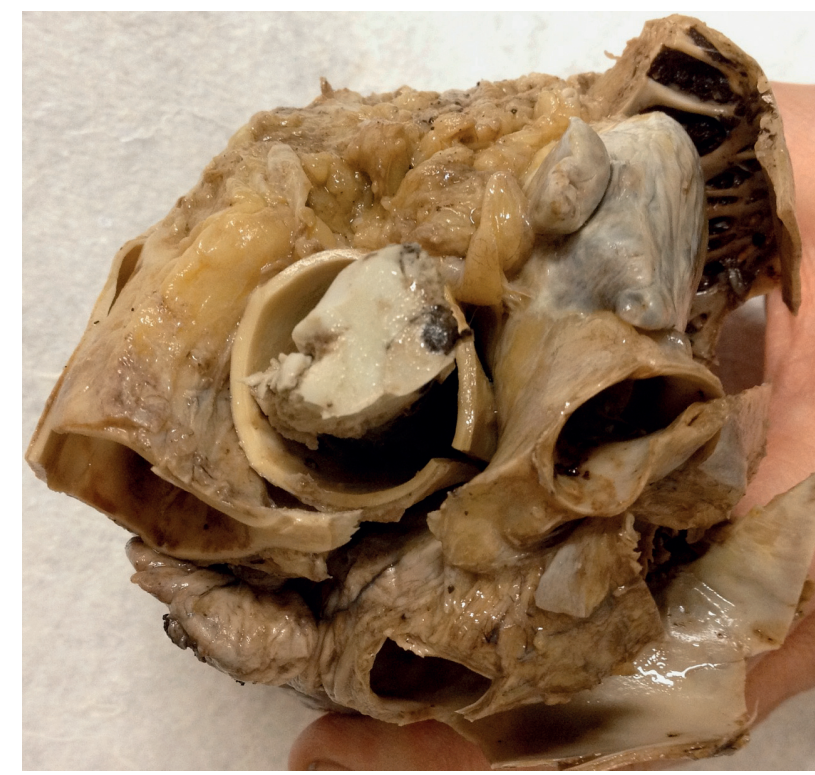

Fig. 1 The mass obstructing the aortic outflow. 
Within the aortic arch, the material was limited to the left side, thereby leaving more space for the blood flow. Distally from the brachiocephalic trunk, only little of this material was found. After removal of this material, a casting of the shape of the sinuses of Valsalva was observed (figure 2).

The heart itself had a normal appearance. None of the 4 cavities were dilated. Hypertrophy and scarring of the myocardium were absent. The aortic valve had three sclerotic but mobile cusps, with small calcified nodules. The left coronary artery was stenotic, but still open. Its major branches as well as the right coronary artery were free of atheromatosis. The aortic arch had a smooth inner surface and its branches were open. Some patchy calcification was observed in the descending aorta but all arteries leading to the brain, the organs and the limbs were open. Furthermore, there were no tumours in the corpse and no scars of major thoraco-abdominal surgery

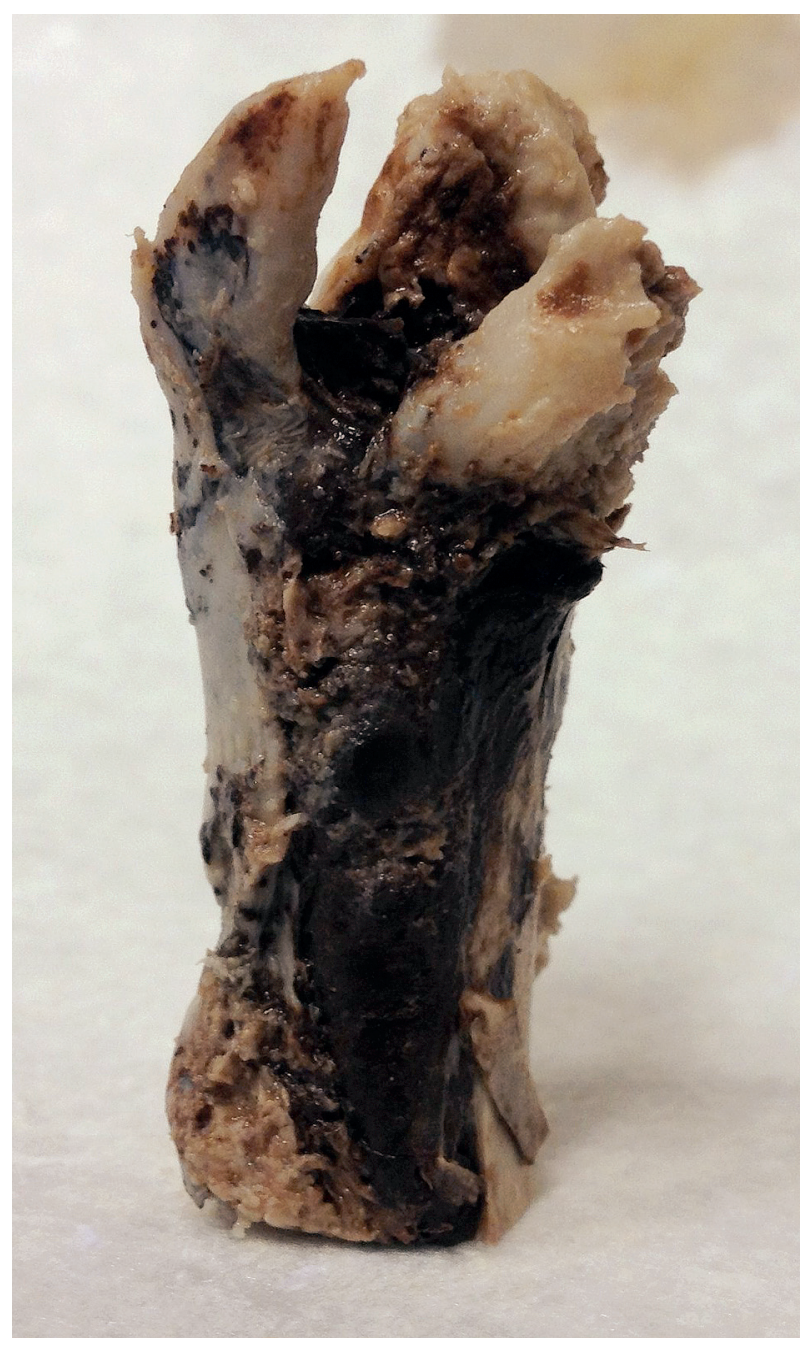

Fig. 2 The cast of the obstructive material with the three extensions into the sinuses of Valsalva. were found. The brain showed no signs of bleeding, and no other causes for mortality were found.

The corpse was embalmed with an aqueous solution of zinc chloride (42\%) containing 1\% Arthyl 26 (Hygeco International, Garges les Gonesse, France). Fixed corpses were stored at $4^{\circ} \mathrm{C}$ before use. After removal of the compact obstruction, this material was thoroughly rinsed in $0.01 \mathrm{M}$ phosphate-buffered saline, and processed for embedding in paraffin. A classic haematoxylin-eosin-staining protocol was used to stain the $7-\mu \mathrm{m}$-thick sections. Microscopic examination showed that the amorphous matter contained fibrinoid material and large cells with multilobed nuclei, characteristic for polymorphonuclear leukocytes.

\section{DISCUSSION}

The material within the ascending aorta probably developed in the last weeks, instead of years before death, which explains the absence of left ventricular hypertrophy. However, left ventricular hypertrophy fails to develop in some patients with aortic valve disease ${ }^{3}$. The observed material extended into the three sinuses of Valsalva, thereby preventing the cusps to open fully. This mimicked the effect of an aortic valve stenosis, and was probably the cause of death, since no other cause for fatality could be found. Sudden death is one of the manifestations of aortic valve obstruction. In this case, it was probably the first one. Infiltration of leukocytes in the fibrinoid material also indicates that the obstruction was already several weeks old. This patient belongs to an age group where aortic valve disease is not uncommon (about 1.3\%), with an average age at onset of stenotic symptoms at 60 years $^{1,2}$. Diagnostic work-up in this case would be more challenging, provided sudden death is not the first sign of this condition: symptoms could evolve more rapidly than in calcified aortic stenosis and signs at auscultation might not be easily interpreted. An echocardiogram with Doppler, necessary for a workup of aortic valve patients ${ }^{4}$, probably would show the restricted motion of the cusps but jet velocity, transvalvular gradient and aortic valve area could be difficult to calculate. Moreover, coronary artery disease is rather common in aortic valve patients. Both diseases share some similarities ${ }^{5,6}$. This condition, however, cannot be documented by coronary angiography in such cases, since the coronary ostiae could not be reached. A cardio$\mathrm{CT}$ and MRI become indispensable. Imaging of the heart, the aortic valve and the full aortic arch would be required to determine the surgical approach in cases where such deposit of material is suspected. The surgical approach itself would also be challenging since installment of an extracorporeal circulation cannot be performed in a classical way. 


\section{CONCLUSION}

This is an unusual case of aortic valve obstruction, which was probably the cause of death. Such diagnosis would require a high degree of suspicion of the attending physician, if the patient survives long enough. The diagnostic workup and the surgical approach will be challenging in such a case of aortic valve obstruction.

\section{ACKNOWLEDGEMENT}

The authors wish to thank the technical staff of the Laboratory of Human Anatomy and Embryology of the University of Antwerp.

CONFLICTS OF INTEREST: none.

\section{REFERENCES}

1. Carabello BA, Paulus WJ. Aortic stenosis. Lancet 2009; 373: 956-66.

2. Baumgartner $\mathrm{H}$, Hung J, Bermejo J, Chambers JB, Evangelista A, Griffin BP, lung B, Otto CM, Pellikka PA, Quinones $M_{i}$ EAE/ASE. Echocardiographic assessment of valve stenosis: EAE/ASE recommendations for clinical practice.

Eur J Echocardiogr 2009; 10: 1-25.
3. Gunther S, Grossman W. Determinants of ventricular function in pressure-overload hypertrophy in man. Circulation 1979; 59: 679-88.

4. Ross J Jr, Braunwald E. Aortic stenosis. Circulation 1968; 38(1suppl): 61-7.

5. Mistiaen W, Van Cauwelaert $P$, Muylaert $P_{,}$ De Worm E. One thousand CarpentierEdwards pericardial valves in the aortic position: what has changed in the past 20 years, and what are the effects on hospital complications? $J$ Heart Valve Dis 2007; 16: 417-22.

6. Somers P, Knaapen M, Kockx M, Bortier $\mathrm{H}_{\text {, }}$ Van Cauwelaert P, Mistiaen W. Histological evaluation of autophagic cell death in calcified aortic valve stenosis. J Heart Valve Dis 2006; 15: 43-8. 